Research article Open Access

\title{
Predicting a local recurrence after breast-conserving therapy by gene expression profiling
}

\author{
Dimitry SA Nuyten ${ }^{1,2,3}$, Bas Kreike ${ }^{1,2,3}$, Augustinus AM Hart ${ }^{1}$, Jen-Tsan Ashley Chi ${ }^{4}$, \\ Julie B Sneddon 4 , Lodewyk FA Wessels², Hans J Peterse ${ }^{2}$, Harry Bartelink ${ }^{1}$, Patrick O Brown ${ }^{4,5}$, \\ Howard Y Chang ${ }^{5}$ and Marc J van de Vijver ${ }^{2}$
}

\author{
1Department of Radiation Oncology, The Netherlands Cancer Institute/Antoni van Leeuwenhoek Hospital, Plesmanlaan 121, 1066 CX Amsterdam, \\ The Netherlands \\ 2Department of Diagnostic Oncology, The Netherlands Cancer Institute/Antoni van Leeuwenhoek Hospital, Plesmanlaan 121, 1066 CX Amsterdam, \\ The Netherlands \\ ${ }^{3}$ Department of Experimental Therapy, The Netherlands Cancer Institute/Antoni van Leeuwenhoek Hospital, Plesmanlaan 121, 1066 CX Amsterdam, \\ The Netherlands \\ 4Department of Biochemistry, Stanford University School of Medicine, Stanford, CA 94305, USA \\ ${ }^{5}$ Program in Epithelial Biology, Howard Hughes Medical Institute, Stanford University School of Medicine, Stanford, CA 94305, USA
}

Corresponding author: Marc J van de Vijver, m.vd.vijver@nki.nl

Received: 11 Apr 2006 Revisions requested: 3 Jul 2006 Revisions received: 14 Sep 2006 Accepted: 30 Oct 2006 Published: 30 Oct 2006

Breast Cancer Research 2006, 8:R62 (doi:10.1186/bcr1614)

This article is online at: http://breast-cancer-research.com/content/8/5/R62

(c) 2006 Nuyten et al.; licensee BioMed Central Ltd.

This is an open access article distributed under the terms of the Creative Commons Attribution License (http://creativecommons.org/licenses/by/2.0), which permits unrestricted use, distribution, and reproduction in any medium, provided the original work is properly cited.

\begin{abstract}
Introduction To tailor local treatment in breast cancer patients there is a need for predicting ipsilateral recurrences after breastconserving therapy. After adequate treatment (excision with free margins and radiotherapy), young age and incompletely excised extensive intraductal component are predictors for local recurrence, but many local recurrences can still not be predicted. Here we have used gene expression profiling by microarray analysis to identify gene expression profiles that can help to predict local recurrence in individual patients.
\end{abstract}

Methods By using previously established gene expression profiles with proven value in predicting metastasis-free and overall survival (wound-response signature, 70-gene prognosis profile and hypoxia-induced profile) and training towards an optimal prediction of local recurrences in a training series, we establish a classifier for local recurrence after breast-conserving therapy.

Results Validation of the different gene lists shows that the wound-response signature is able to separate patients with a high $(29 \%)$ or low $(5 \%)$ risk of a local recurrence at 10 years (sensitivity $87.5 \%$, specificity $75 \%$ ). In multivariable analysis the classifier is an independent predictor for local recurrence.

Conclusion Our findings indicate that gene expression profiling can identify subgroups of patients at increased risk of developing a local recurrence after breast-conserving therapy.

\section{Introduction}

Breast-conserving therapy $(\mathrm{BCT})$ is a well-established treatment modality for early (stages I and II) breast cancer. The treatment consists of complete surgical excision of the tumor followed by whole breast irradiation. In multiple randomized trials comparing BCT with mastectomy, their equality in overall survival has been shown [1-7]. A large population-based Danish series showed that in different age categories (less than 35 years, 35 to 39 years, 40 to 44 years and 45 to 49 years) of young patients, survival was not negatively influenced by BCT
[8]. However, local recurrence rates were significantly higher after BCT than after mastectomy in all series. The standard treatment for a local recurrence is a salvage mastectomy, which negates the original cosmetic intentions of BCT. More importantly, a recent meta-analysis by the Early Breast Cancer Trialists' Collaborative Group showed a negative impact of a local recurrence on survival [9]. They concluded: 'Differences in local treatment that substantially affect local recurrence rates would, in the hypothetical absence of any other causes of death, avoid about one breast cancer death over the next 15 
years for every four local recurrences avoided, and should reduce 15-year overall mortality.'

Identifying patients at high risk for local recurrence in advance and individualizing treatment in these patients (for example, a higher radiotherapy dose ('boost') or a primary mastectomy) is desirable. Several risk factors for local recurrence have been recognized [10-17]. Margin status, young age, an incompletely excised extensive intraductal component and inadequate radiotherapy dose (boost) have been identified as important risk factors for local recurrence [18]. Adjuvant systemic treatment (chemotherapy or hormonal therapy) is known to reduce the risk of a local recurrence $[12,14,16]$.

Previous studies have shown the ability to predict distantmetastasis-free and overall survival in breast cancer with the use of microarray analysis [19-25]. Mechanisms of recurrence in the breast are not necessarily the same as mechanisms involved in distant metastasis. Theoretically, radioresistance of the tumor cells would be an important factor in local recurrence after BCT but not necessarily in distant metastasis.

Previous analyses of gene expressions patterns in a series of 295 early-stage breast cancer patients have identified gene expression signatures that powerfully predict the risk of distant metastasis and mortality $[22,23,26,27]$. In the present study we used a supervised approach to search for gene expression signatures that predict the risk of local recurrence after BCT in a series of 161 early-stage breast cancer patients.

\section{Materials and methods} Tumor samples and patients

This analysis is based on previously reported gene expression profiles of tumors from a series of 295 stage I and II breast cancer patients treated at the Netherlands Cancer Institute between 1984 and 1995 [23]. All patients were under 53 years of age at the time of diagnosis. For this study all the patients from this series who received BCT $(n=161)$ were selected. BCT consisted of a wide local excision and axillary lymph node dissection followed by whole breast irradiation (median dose $50 \mathrm{~Gy}$, range 50 to $54 \mathrm{~Gy}$; the use of 6,8 or 18 MV photons depending on breast diameter); 144 patients received a boost to a median dose of 15 Gy, 98 patients received a low boost ( 14 to $18 \mathrm{~Gy}$ ), and 46 patients received a higher boost (20 to $26 \mathrm{~Gy}$ ). The boost technique was delivered using ${ }^{192}$ (iridium) implantation (88 patients), electrons (31 patients) or photons (25 patients). Pathological margins were assessed as free of tumor in 134 patients, focal involvement by invasive carcinoma only in 3 patients, focal involvement by ductal carcinoma in situ (DCIS) only in 5 patients, and focal involvement by both DCIS and invasive carcinoma in 4 patients; in 8 patients there was more than focal involvement of the margins by DCIS, or the degree of margin involvement could not be determined with certainty. For four patients there was invasive carcinoma at the margin, but the extent of involve- ment could not be determined with certainty, and for three patients this was the case for both invasive carcinoma and a DCIS component. Of all 27 patients with any extent of involved margins, 2 did not receive a boost, 6 received a low boost and 19 a high boost of radiotherapy. Follow-up assessment was at least yearly, including mammography. Seventeen patients developed a local recurrence; the resection margins of the primary tumor had been free of any tumor for 15 of these 17 patients. One patient who later developed a local recurrence had focal involvement of the resection margins with invasive carcinoma, and one patient had focal involvement with DCIS. The 10-year local-recurrence-free percentage was $85 \%$ (median follow-up for all patients was 7.7 years, and 8.5 years for alive patients).

On the basis of the microarray identification number, patients were alternately assigned to the training set (that is, developing a predictor of local recurrence) or to the validation set. This was done separately for locally recurring and locally tumor-free patients, thereby creating almost equal-sized sets with balancing for local recurrence.

Patient characteristics for both the training and validation series are equally divided and are shown in Table 1. In Table 2 characteristics are listed for patients with and without a local recurrence. A full clinical data sheet is available online as Additional file 1, on [28] and on the paper's home page [29].

\section{Microarray procedures}

RNA isolation, labeling of complementary RNA, and hybridization to 25,000-element oliogonucleotide microarrays, and measurement of expression ratios were performed as described previously [23].

The microarray data for analysis of the 70-gene prognosis profile were obtained with the use of the same 25,000-element oligonucleotide array; the correlation coefficient of the expression of these 70 genes with the good prognosis profile was calculated as reported previously [22]. The two other gene lists used are derived from Stanford cDNA arrays [30]. The correspondence between genes represented by Stanford cDNA microarrays and Rosetta/NKI oligonucleotide microarrays were established by using Unigene identifiers (build 158, release date 18 January 2003 for the wound signature, as this was the original mapping carried out for the validation of the wound signature [26]). The primary hypoxia analysis was performed at a later time. We used build 172, release date 17 July 2004 [27]. Because of the relatively small number of genes, probes on the Rosetta/NKI array that were mapped to the same unigene cluster were averaged.

The entire expression database for all 295 patients is publicly available online [28], as are the files containing the expression ratios for the specific experiments (70 genes (Additional file 2), 
Table 1

\begin{tabular}{|c|c|c|c|}
\hline Characteristic & Training & Validation & All \\
\hline No. of patients & 81 & 80 & 161 \\
\hline Non-local recurrence & $72(89 \%)$ & $72(90 \%)$ & $144(89 \%)$ \\
\hline Local recurrence & $9(11 \%)$ & $8(10 \%)$ & $17(11 \%)$ \\
\hline Tumor size $\mathrm{T} 1 / \mathrm{T} 2$ & $50 / 31(62 \% / 38 \%)$ & $48 / 32(60 \% / 40 \%)$ & 98/63 (61\%/39\%) \\
\hline $\mathrm{pNO} / \mathrm{pN}+$ & 46/35 (57\%/43\%) & $44 / 36(55 \% / 45 \%)$ & $90 / 71(56 \% / 44 \%)$ \\
\hline Grade I/II/III & $22 / 26 / 33(27 \% / 32 \% / 41 \%)$ & $21 / 28 / 31(26 \% / 35 \% / 39 \%)$ & $43 / 54 / 64$ (27\%/33\%/40\%) \\
\hline Age $<40$ years & $62(77 \%)$ & $64(80 \%)$ & 126 \\
\hline Follow-up (years) & 7.87 & 7.56 & 7.69 \\
\hline Metastasis & $26(32 \%)$ & $23(29 \%)$ & $49(30 \%)$ \\
\hline Chemotherapy & $28(35 \%)$ & $30(38 \%)$ & $58(36 \%)$ \\
\hline Hormonal therapy & $10(12 \%)$ & $8(10 \%)$ & $18(11 \%)$ \\
\hline $\mathrm{ER}^{+} / \mathrm{ER}-$ & $66 / 15(81 \% / 19 \%)$ & $55 / 25(69 \% / 31 \%)$ & $121 / 40(75 \% / 25 \%)$ \\
\hline Median RT dose (Gy) & 50 & 50 & 50 \\
\hline Median boost dose (Gy) & 15 & 15 & 15 \\
\hline Boost, yes/no & $70 / 11(86 \% / 16 \%)$ & $74 / 6(93 \% / 7 \%)$ & 144/17 (89\%/11\%) \\
\hline Margins infiltrating carcinoma ${ }^{a}$ & $73,4,4(90 \%, 5 \%, 5 \%)$ & $72,3,5(90 \%, 4 \%, 6 \%)$ & $145,7,9(90 \%, 4 \%, 6 \%)$ \\
\hline Margins DCIS b & $25,48,3,5(31 \%, 59 \%, 4 \%, 6 \%)$ & $27,41,7,5(34 \%, 51 \%, 9 \%, 6 \%)$ & $52,89,10,10(33 \%, 55 \%, 6 \%, 6 \%)$ \\
\hline
\end{tabular}

pNO, pathologically node negative; $\mathrm{pN}+$, pathologically node positive; ER, estrogen receptor; RT, radiotherapy; DCIS, ductal carcinoma in situ. aResults are given in the following sequence: radical, focally positive resection margin, extensively positive resection margin or positive resection margin not otherwise specified; bresults are given in the following sequence: no DCIS, radical, focally positive resection margin, extensively positive resection margin or positive resection margin not otherwise specified.

hypoxia signature (Additional file 3 ) and wound signature (Additional file 4) [29].

\section{Class prediction and gene selection de novo}

The first method we used for predicting local recurrence is PAM (prediction analysis for microarrays): class prediction as described by Tibshirani and colleagues [31]. This method shifts the mean expression level of each gene (possibly after transformation) for each class (for example local recurrence) towards the overall mean expression level for all classes by a fixed standardized difference (shrunken centroids). The class for which the shrunken centroid most closely reaches the observed expression pattern of a certain patient by using a Pearson correlation is then the predicted class for that patient. For a given shrunken centroid only those genes for which the shrunken means still differ from the overall mean will contribute to the distance between centroids and any individual tumor sample's expression pattern. The standardized difference, and thereby the number of relevant genes, is chosen by minimizing the prediction error using 10-fold balanced, leave-10\%-out cross-validation within the training set. The same method is then used to predict classes for new samples (validation set).

\section{Gene set enrichment analysis}

Subramanian and colleagues [32] have described a method that analyzes whether or not predefined gene sets (representing biological pathways) are coordinately and significantly upregulated or downregulated between samples that represent different biological or clinical entities [32]. The program corrects for multiple testing. We applied this method to our data set and analyzed gene set C2 ('functional gene set'). The software and gene set are available for download [33]. C2 represents 504 gene sets. Standard parameters were used for the analysis. The threshold for false discovery rate is $25 \%$ before a gene set is called significantly upregulated or downregulated.

\section{Established gene expression profiles and methods of supervised analysis}

The following previously established gene expression profiles were used to find a classifier for local recurrence after BCT:

1. The wound-response gene expression signature is a characteristic pattern of expression of a set of genes that have been identified as being induced or repressed in response to 
Breast Cancer Research Vol 8 No 5 Nuyten et al.

Table 2

Clinical characteristics by local recurrence versus non local recurrence

\begin{tabular}{|c|c|c|c|c|}
\hline Characteristic & Non-local recurrence & Local recurrence & $p^{\mathrm{a}}$ & All \\
\hline No. of patients & 144 & 17 & & 161 \\
\hline Tumor size T1/T2 & 86/58 (67\%/33\%) & $12 / 5(71 \% / 29 \%)$ & 0.46 & $98 / 63(61 \% / 39 \%)$ \\
\hline $\mathrm{pNO} / \mathrm{pN}+$ & $83 / 61(58 \% / 42 \%)$ & $7 / 10(41 \% / 59 \%)$ & 0.19 & $90 / 71(56 \% / 44 \%)$ \\
\hline Grade $1 / I I / I I I$ & $41 / 47 / 56(28 \% / 33 \% / 39 \%)$ & 2/7/8 (12\%/41\%/47\%) & 0.16 & 43/54/64 (27/33/40\%) \\
\hline Age $<40$ years & $28(19 \%)$ & $7(41 \%)$ & 0.074 & 35 \\
\hline Median follow-up (years) & 7.68 & 9.31 & 0.014 & 7.69 \\
\hline Metastasis & $40(28 \%)$ & $9(53 \%)$ & 0.004 & $49(30 \%)$ \\
\hline Chemotherapy & $50(35 \%)$ & $8(47 \%)$ & 0.291 & $58(36 \%)$ \\
\hline Hormonal therapy & $16(11 \%)$ & $2(12 \%)$ & 0.86 & $18(11 \%)$ \\
\hline $\mathrm{ER}^{+} / \mathrm{ER}-$ & $109 / 35(76 \% / 24 \%)$ & $12 / 5(71 \% / 29 \%)$ & 0.42 & $121 / 40(75 \% / 25 \%)$ \\
\hline Median RT dose (Gy) & 50 & 50 & 0.47 & 50 \\
\hline Median boost dose (Gy) & 15 & 15 & 0.22 & 15 \\
\hline Boost, yes/no & $131 / 13(91 \% / 9 \%)$ & $13 / 4(76 \% / 24 \%)$ & 0.075 & $144 / 17(89 \% / 11 \%)$ \\
\hline Margins infiltrating carcinoma ${ }^{b}$ & $129,6,9(90 \%, 4 \%, 6 \%)$ & $16,1,0(94 \%, 6 \%, 0 \%)$ & 0.365 & $145,7,9(90 \%, 4 \%, 6 \%)$ \\
\hline Margins DCISc & $49,76,9,10(34 \%, 53 \%, 6 \%, 7 \%)$ & $3,13,1,0(18 \%, 76 \%, 6 \%, 0 \%)$ & 0.65 & $52,89,10,10(33 \%, 55 \%, 6 \%, 6 \%)$ \\
\hline
\end{tabular}

pNO, pathologically node negative; pN+, pathologically node positive; ER, estrogen receptor; RT, radiotherapy; DCIS, ductal carcinoma in situ. ${ }^{\mathrm{a} C o x}$ univariate; bresults are given in the following sequence: radical, focally positive resection margin, extensively positive resection margin or positive resection margin not otherwise specified; cresults are given in the following sequence: no DCIS, radical, focally positive resection margin extensively positive resection margin or positive resection margin not otherwise specified.

bovine serum, a surrogate wound environment [30,34]. This so-called 'core serum response' has shown to be a strong predictive factor for outcome in cancer (breast, head and neck, lung and gastric) using metastasis-free and overall survival as endpoints $[26,30]$. It is important to realize that a particular pattern of expression of these genes, not just the genes themselves, predicts outcome.

2. The 70-gene prognosis profile was originally trained to predict metastasis within 5 years ('poor prognosis' group), or no metastasis within 5 years ('good prognosis' group) [22].

3. The hypoxia profile is a gene expression classifier from an in vitro experiment in which epithelial cells are exposed to hypoxia [27].

First, these gene expression profiles were used in their original form and tested for their value in predicting local recurrence after BCT in the complete set of 161 patients.

Second, we optimized these profiles by using the clinical data on local recurrence.

Using the training data set, we calculated for each of the three profiles the average pattern of expression of the genes that comprised each profile, for patients who developed a local recurrence (local recurrence profile or local recurrence centroid). For all patients in the training and validation set we then calculated the non-centralized Pearson correlation to each of the three average patterns of expression (local recurrence profile or local recurrence centroid). A correlation value between -1 and +1 is thereby derived for all patients and indicates the similarity in gene expression between an individual patient (tumor) and the average gene expression of tumors from patients in the training set who developed a local recurrence.

For each of the three signatures a plot of these correlation values (see Figure 1 for the core serum response genes) is drawn for the training set and shows the difference in expression between patients who developed a local recurrence and those who did not. On the basis of the difference in the distribution of these correlation values, we selected the maximal threshold, misclassifying only one out of nine local recurrences in the training set. Patients with a correlation value above the threshold were classified as high-risk, and patients below the threshold as low-risk. Except for the gene selection process, this is the same procedure as followed by van't Veer and colleagues [21]. The local recurrence profile and its associated threshold value were subsequently validated on the validation set. The 
methods for training and validation are depicted schematically in Figure 2.

\section{Statistics}

All patients who developed a local recurrence were considered; 16 patients developed a local recurrence as a first event and in 1 patient local recurrence occurred concurrently with distant metastases. Local-recurrence-free percentages were calculated with the Kaplan-Meier method (log-rank test for comparison). All Kaplan-Meier curves are available as supplementary data in Additional file 5. Comparison of categorical variables between groups was performed with a $\chi^{2}$ test. For the multivariable analysis we used a Cox regression model. All $p$ values are two-sided. All correlations were calculated by the non-centralized Pearson method. For analysis we used Winstat ${ }^{\circledR}$ for Excel (R. Fitch Software, Staufen, Germany), SPSS 13.0 ${ }^{\circledR}$ (SPSS Inc., Chicago, IL, USA) and Microsoft Excel ${ }^{\circledR}$ (Microsoft Corporation) were used. The PAM analysis was performed with version 1.2 (November 2003) [31,35].

Additional material and methods are available described in Additional file 9.

\section{Results}

In a previously reported series of 295 patients with stage I and Il breast cancer diagnosed at age less than 53 years, 161 had been treated with BCT (the remaining 134 patients underwent modified radical mastectomy). Of these 161 patients, 17 developed a local recurrence. From the series of 161 patients, a training and validation set were selected as described in the Materials and methods section, distributing local recurrences equally. This resulted in a balanced distribution of year of treat-

\section{Figure 1}

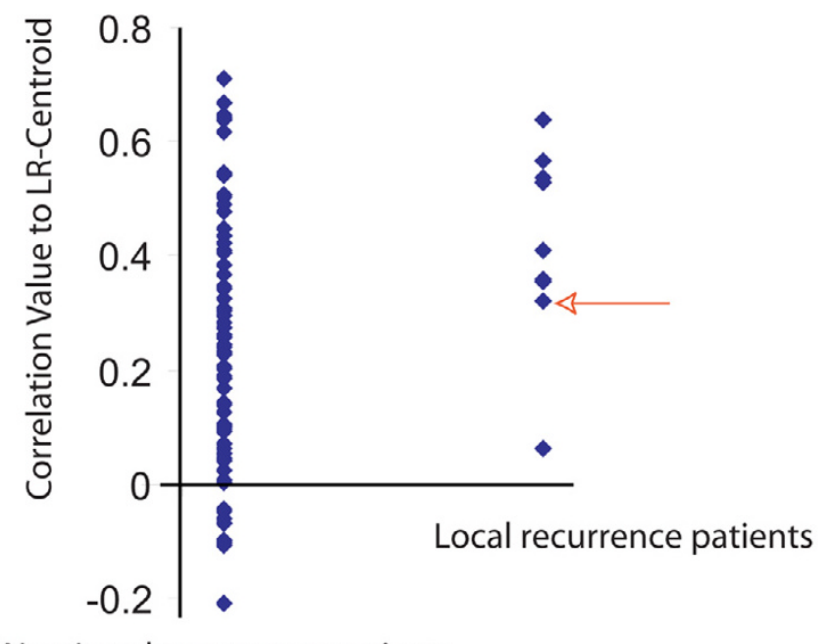

Non Local recurrence patients

Local recurrence Wound-signature correlation plot for the training set. Red arrow indicates optimal threshold. ment and major risk factors for local recurrence between the two groups as well (Table 1).

\section{Training and validation series}

Nine out of 81 patients in the training set had developed a local recurrence at a median interval of 6.2 years (range 1.0 to 10.8 years); the median follow-up time for the patients in the entire training set was 7.9 years. Six out of nine local recurrence patients had received a boost (five patients received 15 Gy, one patient 25 Gy).

In the validation set, 8 out of 80 patients had developed a local recurrence at a median interval of 3.72 years (range 1.2 to 8.4 years); the median follow-up time for all patients in the validation set was 7.6 years. Seven out of these eight patients had received a boost (three patients received 15 Gy, four patients 25 Gy).

\section{Can a local recurrence classifier be identified by supervised classification using all genes?}

We used the PAM algorithm with the training data set to search for gene expression profiles predictive of local recurrence, starting with a gene list that consisted of the top 5,000 most variably expressed genes with technically well-measured expression, represented by microarrays (out of 25,000). The performance of this approach, as evaluated by cross-validation, seemed to be poor: only 4 out of 9 local recurrences and 50 of the 72 non-local recurrences could be predicted accurately in the training set at this threshold (using a profile based on 684 genes). The use of fewer genes resulted in more accurate prediction for non-local-recurrence patients, but less accuracy for local-recurrence patients. In the validation series only 1 out of 8 local recurrences and 65 out of 72 non-local recurrences were predicted correctly (PAM analysis is provided in Additional files 6, 7, 8).

\section{Using predefined gene sets based on biological processes: gene set enrichment analysis}

We used the GSEA (gene set enrichment analysis) software to analyze whether or not gene sets defined on the basis of biological processes or pathways were significantly correlated with local recurrence. We first ran the analysis on the full probe set (more than 23,000). We than ran the analysis on the same selection of genes used for the PAM analysis (the top 5,000). Neither the full probe set nor the top 5,000 probe set revealed any gene set to be significantly upregulated or downregulated in samples from tumors that recurred locally, after correction for multiple testing.

\section{Using previously identified gene expression signatures to predict local recurrence}

We have previously shown that three distinct gene expression signatures can each be used to subdivide breast carcinomas into two groups that are associated, respectively, with good and poor distant-metastasis-free and overall survival: a '70- 


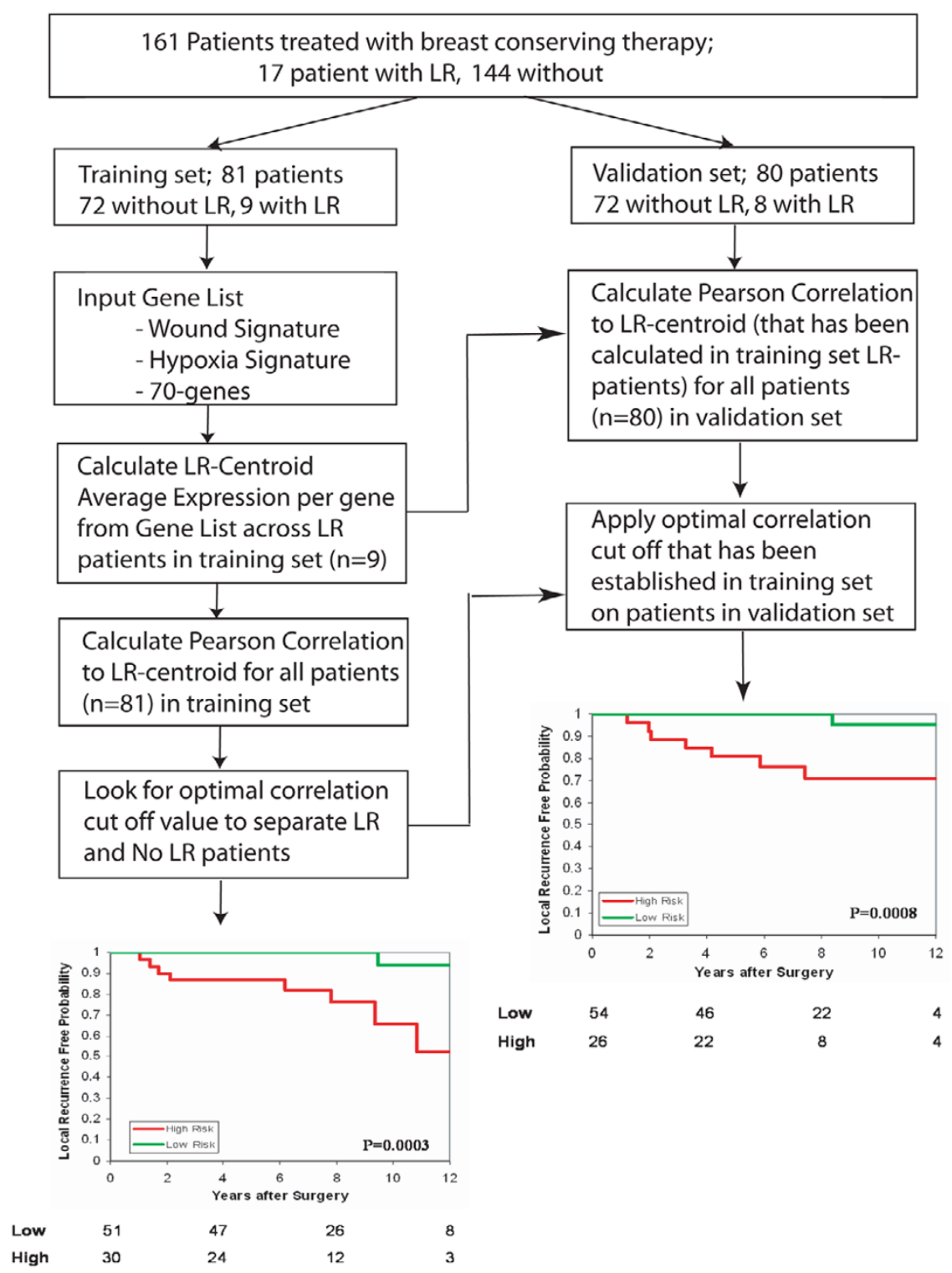

Scheme for training and validation, including Kaplan-Meier curves for local-recurrence-free survival.

gene' prognosis profile [22,23], a wound-response signature $[26,30]$ and a hypoxia-response signature [27]. We tested whether these three signatures were also associated with a difference in the risk of local recurrence. The 70-gene prognosis profile was originally trained to predict metastasis within 5 years ('poor prognosis' group) or no metastasis within 5 years ('good prognosis' group); in the current series of 161 patients, 97 patients had tumors with a poor prognosis profile and 64 patients had tumors with a good prognosis profile.

The wound-response signature was derived from an in vitro model for wound healing and can be used to classify tumors as having an 'activated wound-response signature' or a 'quiescent wound-response signature' $[26,30]$. In this series of tumors, 380 (out of 459) genes from the wound-response signature were identified; 65 tumors had an activated woundresponse signature and 96 tumors had a quiescent woundresponse signature.

The hypoxia-response profile is a gene expression classifier derived from an ex vivo model in which epithelial cells are exposed to hypoxia [27]. In this series of tumors, 123 (out of 168) genes from the hypoxia-response signature were identified. On the basis of the expression of these hypoxia-associ- 
ated genes, the breast carcinomas in this study could be assigned to 36 cases with a hypoxia-response signature and 125 with a non-hypoxia signature.

Of these three signatures, only the wound-response signature showed some evidence ( $p=0.04$; log-rank) of predicting local recurrence (13\% versus $19 \%$ at 10 years for quiescent versus activated).

\section{Building a classifier for local recurrence based on existing gene selections}

Because the supervised approach using all genes (class prediction; PAM) failed and the previously identified gene expression signatures could not convincingly predict the risk of a local recurrence, we used genes from the original profiles and constructed a new classifier based on the relationship of their expression patterns to clinical outcome observed in our study (local recurrence or no local recurrence).

Each of these three signatures was originally defined for purposes other than the prediction of local recurrence, but we hypothesized that the optimization of each signature's pattern threshold by training on local recurrence data might improve their performance for this new purpose.

\section{Seventy-gene prognosis profile}

Applying the method described in the Materials and methods section, a correlation value of 0.2364 was calculated as a cutoff point for the 70-gene expression profile as a predictor of local recurrence, resulting in one misclassified patient with local recurrence. The recurrence risks at 10 years were $7 \%$ and $28 \%$ for the low-risk and high-risk groups, respectively.

In the validation set the separation could not be reproduced ( $p$ $=0.34$; log-rank). The sensitivity was $63 \%$ (5 out of 8 ) and the specificity $50 \%$ (36 out of 72 ). At 10 years the recurrence risk was $13 \%$ versus $14 \%$ for the low-risk group versus the highrisk group. Furthermore, more than half the patients were assigned by this classifier to the high-risk group (41/80).

\section{Hypoxia-response signature}

For the hypoxia-response gene expression signature, the threshold that misses one patient with local recurrence seemed to be 0.254 . In the training set the estimated 10-year recurrence risks for the low-risk and high-risk groups as determined by using this threshold were $10 \%$ and $19 \%$, respectively. The performance of this signature on the validation set was not significant $(p=0.4)$. By employing this threshold on the validation set, 46 patients $(58 \%)$ were classified as 'high risk' and 34 (42\%) as low risk. At 10 years the recurrence risk was $13 \%$ versus $15 \%$ for low-risk versus high-risk, respectively. The sensitivity and specificity were $75 \%$ (6 out of 8 ) and $44 \%$ (32 out of 72 ), respectively.

\section{Wound-response signature: core serum response genes} The wound-response signature was first identified as a pattern of changes in gene expression induced by exposing fibroblasts to serum. A threshold value of 0.3179 for the correlation with the wound-response signature (as derived from the 380 available genes) resulted in one missed local recurrence in the training set. With the use of the threshold value for classification, 30 patients (37\%) were classified as high-risk, and 51 $(63 \%)$ as low-risk. In the training set, the 10-year recurrence risk was $6 \%$ for the 'low-risk' group and 35\% for the 'high-risk' group. With this threshold, in the validation set 26 patients (33\%) were classified as high-risk, and 54 (67\%) as low-risk. The 10-year recurrence risk in the validation set was $5 \%$ for the low-risk group, in contrast with $29 \%$ for the high-risk group ( $p=0.0008$; log-rank). The predictive performance of the wound-response local recurrence signature on the validation set, using the threshold derived from the training set, is shown in Figure 2. The sensitivity of this classifier for predicting local recurrence in the validation set was $88 \%$ (7 out of 8 ) with a specificity of $74 \%$ (53 out of 72 ). The hazard ratio was 15 (95\% confidence interval (Cl) 1.8 to 123 ; log-rank 0.01). An overview of the sensitivity and specificity for the three supervised approaches is shown in Table 3.

\section{Cox regression: multivariable analysis}

To assess the independent prognostic value of the supervised wound-response signature, it was tested in a Cox regression model that also included known clinico-pathological risk fac-

Table 3

Performance of three different supervised analyses on validation series

\begin{tabular}{llll}
\hline Analysis & WS supervised & 70 -genes supervised & Hypoxia supervised \\
\hline Sensitivity (percentage) & 88 & 63 & 75 \\
Specificity (percentage) & 74 & 50 & 44 \\
Low risk/high riska (percentage) & $67 / 33$ & $49 / 51$ & $42 / 58$ \\
10-year LC low/high (percentage) & $95 / 71$ & $87 / 86$ & $87 / 85$ \\
$p^{\text {b }}$ & 0.0008 & 0.34 & 0.4 \\
\hline
\end{tabular}

WS, wound signature; LC, local control.

aln validation set (percentages; $n=80$ ); ${ }^{p} p$ value in validation set (log-rank). 
tors for local recurrence, using the independent validation data set. The factors that independently predicted a local recurrence in multivariable analysis in the EORTC 'boost versus no boost' trial were tested together with the local recurrence classifiers [17]. In the presence of age, diameter and boost treatment, all the gene expression classifiers were tested. Only the wound-response local recurrence signature (average local recurrence centroid) added significant prognostic information to the classical risk factors in predicting a local recurrence (hazard ratio $16(95 \% \mathrm{Cl} 1.9$ to 125$) ; p=0.01)$. Further results of the multivariable analysis are shown in Table 4.

\section{Discussion}

We have shown that gene expression profiling using DNAmicroarray analysis can be used in risk stratification for local recurrence after BCT in breast cancer patients. We tested several different methods: standard supervised analysis (PAM and GSEA, with local recurrence as the end point), profiles that have previously been used to predict distant metastasis and survival, and a combination of both approaches by supervising these previously established predictive profiles with clinical data on local recurrence.

The supervised wound-response signature derived by this approach is the only profile that could predict a local recurrence after BCT. This new method begins with a biologically defined gene set and constructs a classifier by a supervised learning procedure from the expression pattern of the selected gene set, using clinical data. This thereby uses not only the genes themselves but also the expression levels for the different genes in the signature that are correlated with the clinical end point (local recurrence). The power of this signature in predicting local recurrence was highly significant, even if we conservatively take into account the fact that we tried a total of seven methods, by applying a Bonferroni correction to the $p$ value of 0.0008 associated with the supervised wound signature. It is important to note that the gene set derived from an in vitro model, the wound response, but not the wound-response gene expression signature itself, was adapted to derive a gene expression pattern predictive of a local recurrence in patients who were treated with BCT.
Gene expression profiling has been successfully applied to distinguish molecular subtypes in breast cancer and to predict metastases and overall survival [19-23,25]. More recently, gene expression profiling has also been used in trying to identify signatures predicting response to neo-adjuvant chemotherapy treatment $[36,37]$, and prognosis after treatment with tamoxifen [38-41]. Because many patients are suitable candidates for breast conservation on the basis of low baseline risk for developing a local recurrence, this treatment has become standard for more than $60 \%$ of breast cancer patients. A recently published pooled analysis shows that local recurrence is associated with an increased risk of developing distant metastases and subsequent death from breast cancer [42]. These findings are similar to the recently published EBCTCG (the Early Breast Cancer Trialists' Collaborative Group) meta-analysis [9]. After BCT without radiotherapy, an absolute increase of $5.4 \%$ in breast cancer mortality was seen. Moreover, local recurrence requires treatment (usually salvage mastectomy) and is associated with anxiety for affected patients. It is therefore important to tailor BCT in such a way that the risk of local recurrence is kept as low as possible, while optimizing the quality of life, including the cosmetic appearance of the breast. To select patients who are likely to benefit from BCT, clinico-pathological parameters are currently used to stratify patients by risk of local recurrence. These existing prognostic factors are far from perfect, and additional or better predictors of local recurrence would be of great potential benefit. Aggressive treatment including a larger excision volume and/or a higher local radiotherapy dose ('boost') significantly decreases the risk at of local recurrence but results in impaired cosmetic outcome [43]. In patients considered to be at low risk of local recurrence, the boost dose could be forgone and in patients with a high risk a boost should be administered or even escalated. Furthermore, for patients at very high risk of local recurrence a primary mastectomy can be offered.

Because the number of patients and the number of local recurrence events was low in this study, these results must be interpreted with caution. Although the hazard ratio for a local recurrence in the validation series is high (15), the confidence

Table 4

Cox regression analysis on local recurrence: clinical risk factors and wound signature (validation series only)

\begin{tabular}{|c|c|c|c|c|}
\hline \multirow[t]{2}{*}{ Risk factor } & \multirow[t]{2}{*}{ Significance } & \multirow[t]{2}{*}{ Hazard ratio for local recurrence } & \multicolumn{2}{|c|}{$95 \% \mathrm{Cl}$ for hazard ratio } \\
\hline & & & Lower & Upper \\
\hline Age $<40$ years & 0.39 & 2.542 & 0.30 & 21.7 \\
\hline Tumor size T2/T1 & 0.57 & 1.683 & 0.31 & 9.1 \\
\hline Boost treatment no/yes & 0.83 & 1.343 & 0.09 & 20.2 \\
\hline Wound signature high risk/low risk & 0.01 & 16 & 1.9 & 125 \\
\hline
\end{tabular}

Cl: confidence interval. 
interval is wide (by univariate analysis, 95\% Cl 1.8 to 123 ). Given the potential contribution of this prognostic signature to improve decision making in planning the treatment of breast cancer and an improved understanding of the pathogenesis of local recurrence, an independent test of its predictive value is a high priority. Recently, in The Netherlands, a randomized clinical trial started, to compare the standard radiotherapy dose (50 Gy whole breast and 16 Gy boost to the tumor bed) with an additional boost dose (26 Gy). In this trial, fresh frozen tumor samples will be collected. This trial will allow prospective validation of the signature.

\section{Conclusion}

We have presented a method of integrating biologically derived gene expression profiles and clinical data to build a classifier for local recurrence after breast-conserving therapy. Although numbers of events are small and the resulting confidence intervals are wide, it is the first classifier based on microarray analysis to specifically predict a local recurrence after breast-conserving therapy. In our series the classifier is the most powerful predictor and is independent of clinical and pathological variables.

\section{Competing interests}

The authors declare that they have no competing interests.

\section{Authors' contributions}

DSAN conceived the method of building the gene expression profile specifically for local recurrence and performed the primary analysis. DSAN, BK, AAMH, JTAC, JBC, LFW, HJP, HB, $\mathrm{POB}, \mathrm{HYC}$ and MJvdV analyzed and interpreted data. DSAN, AAMH and MJvdV wrote the paper. All authors read and approved the final manuscript.

\section{Additional files}

The following Additional files are available online:

\section{Additional file 1}

An Excel file containing all clinical data, including all labels for various classifiers (both the original previously published labels as well as the local recurrence labels), and the correlation values (to the local recurrence centroids) for all patients.

See http://www.biomedcentral.com/content/ supplementary/bcr1614-S1.xls

\section{Additional file 2}

An Excel file containing the expression data for the 70 genes for all patients (training (local recurrence and nonlocal recurrence) and validation set separately) and the calculated 70 genes local recurrence centroid data and correlation values for all patients to this centroid.

See http://www.biomedcentral.com/content/ supplementary/bcr1614-S2.xls

\section{Additional file 3}

An Excel file containing the expression data for the hypoxia genes for all patients (training (local recurrence and non-local recurrence) and validation set separately) and the calculated hypoxia local recurrence centroid data and correlation values for all patients to this centroid.

See http://www.biomedcentral.com/content/ supplementary/bcr1614-S3.xls

\section{Additional file 4}

An Excel file containing the expression data for the wound signature genes for all patients (training (local recurrence and non local recurrence) and validation set separately) and the calculated wound signature local recurrence centroid data and correlation values for all patients to this centroid.

See http://www.biomedcentral.com/content/ supplementary/bcr1614-S4.xls

\section{Additional file 5}

An Excel file containing all Kaplan-Meier curves for the original classification and the supervised classifications. See http://www.biomedcentral.com/content/ supplementary/bcr1614-S5.xls

\section{Additional file 6}

A text file containing the expression data for the 5,000 most variable expressed genes on the array for all patients in the training set.

See http://www.biomedcentral.com/content/ supplementary/bcr1614-S6.txt

\section{Additional file 7}

A text file containing the expression data for the 5,000 most variable expressed genes on the array for all patients in the validation set.

See http://www.biomedcentral.com/content/ supplementary/bcr1614-S7.txt

\section{Additional file 8}

An Excel file containing the results from the PAM analysis.

See http://www.biomedcentral.com/content/ supplementary/bcr1614-S8.xls

\section{Additional file 9}

A Word file containing supplementary additional details of material and methods.

See http://www.biomedcentral.com/content/ supplementary/bcr1614-S9.doc 


\section{Acknowledgements}

This work was supported by Dutch Cancer Society Grant NKB 20022575 (to DSAN, BK, HB and MJvdV), National Institutes of Health Grants CA77097 and CA85129 (to POB) and AR050007 (to HYC), and a National Science Foundation Predoctoral Fellowship (to JBS). $\mathrm{POB}$ is an Investigator of the Howard Hughes Medical Institute.

\section{References}

1. Sarrazin D, Le MG, Arriagada R, Contesso G, Fontaine F, Spielmann M, Rochard F, Le Chevalier T, Lacour J: Ten-year results of a randomized trial comparing a conservative treatment to mastectomy in early breast cancer. Radiother Oncol 1989, 14:177-184.

2. Blichert-Toft M, Rose C, Andersen JA, Overgaard M, Axelsson CK, Andersen KW, Mouridsen HT: Danish randomized trial comparing breast conservation therapy with mastectomy: six years of life-table analysis. Danish Breast Cancer Cooperative Group. J Natl Cancer Inst Monogr 1992, 11:19-25.

3. Veronesi U, Marubini E, Mariani L, Galimberti V, Luini A, Veronesi $\mathrm{P}$, Salvadori B, Zucali R: Radiotherapy after breast-conserving surgery in small breast carcinoma: long-term results of a randomized trial. Ann Oncol 2001, 12:997-1003.

4. Mariani L, Salvadori B, Marubini E, Conti AR, Rovini D, Cusumano F, Rosolin T, Andreola S, Zucali R, Rilke F, Veronesi U: Ten year results of a randomised trial comparing two conservative treatment strategies for small size breast cancer. Eur J Cancer 1998, 34:1156-1162.

5. Veronesi U, Salvadori B, Luini A, Greco M, Saccozzi R, del Vecchio $M$, Mariani L, Zurrida S, Rilke F: Breast conservation is a safe method in patients with small cancer of the breast. Long-term results of three randomised trials on 1,973 patients. Eur $J$ Cancer 1995, 31A:1574-1579.

6. van Dongen JA, Voogd AC, Fentiman IS, Legrand C, Sylvester RJ, Tong D, van der SE, Helle PA, van Zijl K, Bartelink H: Long-term results of a randomized trial comparing breast-conserving therapy with mastectomy: European Organization for Research and Treatment of Cancer 10801 trial. J Natl Cancer Inst 2000, 92:1143-1150.

7. Fisher B, Anderson S, Bryant J, Margolese RG, Deutsch M, Fisher ER, Jeong $\mathrm{JH}$, Wolmark N: Twenty-year follow-up of a randomized trial comparing total mastectomy, lumpectomy, and lumpectomy plus irradiation for the treatment of invasive breast cancer. N Engl J Med 2002, 347:1233-1241.

8. Kroman N, Holtveg H, Wohlfahrt J, Jensen MB, Mouridsen HT, Blichert-Toft M, Melbye M: Effect of breast-conserving therapy versus radical mastectomy on prognosis for young women with breast carcinoma. Cancer 2004, 100:688-693.

9. Clarke M, Collins R, Darby S, Davies C, Elphinstone P, Evans E, Godwin J, Gray R, Hicks C, James S, et al.: Effects of radiotherapy and of differences in the extent of surgery for early breast cancer on local recurrence and 15-year survival: an overview of the randomised trials. Lancet 2005, 366:2087-2106.

10. Voogd AC, Nielsen M, Peterse JL, Blichert-Toft M, Bartelink $H$, Overgaard M, van Tienhoven G, Andersen KW, Sylvester RJ, van Dongen JA: Differences in risk factors for local and distant recurrence after breast-conserving therapy or mastectomy for stage I and II breast cancer: pooled results of two large European randomized trials. J Clin Oncol 2001, 19:1688-1697.

11. Borger J, Kemperman H, Hart A, Peterse H, van Dongen J, Bartelink $\mathrm{H}$ : Risk factors in breast-conservation therapy. J Clin Oncol 1994, 12:653-660.

12. Elkhuizen $\mathrm{PH}$, van Slooten $\mathrm{HJ}$, Clahsen PC, Hermans J, van de Velde CJ, van den Broek LC, van de Vijver MJ: High local recurrence risk after breast-conserving therapy in node-negative premenopausal breast cancer patients is greatly reduced by one course of perioperative chemotherapy: a European Organization for Research and Treatment of Cancer Breast Cancer Cooperative Group Study. J Clin Oncol 2000, 18:1075-1083.

13. Elkhuizen PH, Voogd AC, van den Broek LC, Tan IT, van Houwelingen $\mathrm{HC}$, Leer JW, van de Vijver MJ: Risk factors for local recurrence after breast-conserving therapy for invasive carcinomas: a case-control study of histological factors and alterations in oncogene expression. Int J Radiat Oncol Biol Phys 1999, 45:73-83.

14. Fredriksson I, Liljegren G, Palm-Sjovall M, Arnesson LG, Emdin $\mathrm{SO}$, Fornander $\mathrm{T}$, Lindgren $\mathrm{A}$, Nordgren $\mathrm{H}$, Idvall I, Holmqvist $\mathrm{M}$, et al.: Risk factors for local recurrence after breast-conserving surgery. Br J Surg 2003, 90:1093-1102.

15. Voogd AC, Peterse JL, Crommelin MA, Rutgers EJ, Botke G, Elkhuizen PH, van Geel AN, Hoekstra CJ, van Pel R, van de Vijver MJ, Coebergh JW: Histological determinants for different types of local recurrence after breast-conserving therapy of invasive breast cancer. Dutch Study Group on local Recurrence after Breast Conservation (BORST). Eur J Cancer 1999, 35:1828-1837.

16. Park CC, Mitsumori M, Nixon A, Recht A, Connolly J, Gelman R, Silver B, Hetelekidis S, Abner A, Harris J, Schnitt S: Outcome at 8 years after breast-conserving surgery and radiation therapy for invasive breast cancer: influence of margin status and systemic therapy on local recurrence. J Clin Oncol 2000, 18:1668-1675.

17. Vrieling C, Collette L, Fourquet A, Hoogenraad WJ, Horiot JC, Jager JJ, Bing Oei S, Peterse HL, Pierart M, Poortmans PM, et al.: Can patient-, treatment- and pathology-related characteristics explain the high local recurrence rate following breast-conserving therapy in young patients? Eur J Cancer 2003, 39:932-944.

18. Bartelink $H$, Horiot JC, Poortmans $P$, Struikmans $H$, Van den Bogaert W, Barillot I, Fourquet A, Borger J, Jager J, Hoogenraad W, et al:: Recurrence rates after treatment of breast cancer with standard radiotherapy with or without additional radiation. N Engl J Med 2001, 345:1378-1387.

19. Perou CM, Sorlie T, Eisen MB, van de Rijn M, Jeffrey SS, Rees CA Pollack JR, Ross DT, Johnsen H, Akslen LA, et al.: Molecular portraits of human breast tumours. Nature 2000, 406:747-752.

20. Sorlie T, Tibshirani R, Parker J, Hastie T, Marron JS, Nobel A, Deng $\mathrm{S}$, Johnsen H, Pesich R, Geisler S, et al:: Repeated observation of breast tumor subtypes in independent gene expression data sets. Proc Natl Acad Sci USA 2003, 100:8418-8423.

21. Sorlie T, Perou CM, Tibshirani R, Aas T, Geisler S, Johnsen H, Hastie T, Eisen MB, van de Rijn M, Jeffrey SS, et al.: Gene expression patterns of breast carcinomas distinguish tumor subclasses with clinical implications. Proc Natl Acad Sci USA 2001, 98:10869-10874.

22. van't Veer $L J$, Dai $H$, van de Vijver MJ, He YD, Hart AA, Mao M, Peterse HL, van der Kooy K, Marton MJ, Witteveen AT, et al.: Gene expression profiling predicts clinical outcome of breast cancer. Nature 2002, 415:530-536.

23. van de Vijver MJ, He YD, van't Veer LJ, Dai H, Hart AA, Voskuil DW, Schreiber GJ, Peterse JL, Roberts C, Marton MJ, et al:: A geneexpression signature as a predictor of survival in breast cancer. N Engl J Med 2002, 347:1999-2009.

24. Sotiriou C, Neo SY, McShane LM, Korn EL, Long PM, Jazaeri A, Martiat P, Fox SB, Harris AL, Liu ET: Breast cancer classification and prognosis based on gene expression profiles from a population-based study. Proc Natl Acad Sci USA 2003, 100:10393-10398.

25. Wang $Y$, Klijn JG, Zhang $Y$, Sieuwerts AM, Look MP, Yang F, Talantov D, Timmermans M, Meijer-van Gelder ME, Yu J, et al: Gene-expression profiles to predict distant metastasis of lymph-node-negative primary breast cancer. Lancet 2005, 365:671-679.

26. Chang HY, Nuyten DS, Sneddon JB, Hastie T, Tibshirani R, Sorlie $T$, Dai H, He YD, van't Veer LJ, Bartelink $H$, et al:: Robustness, scalability, and integration of a wound-response gene expression signature in predicting breast cancer survival. Proc Nat/ Acad Sci USA 2005, 102:3738-3743.

27. Chi JT, Wang Z, Nuyten DS, Rodriguez EH, Schaner ME, Salim A, Wang $Y$, Kristensen GB, Helland A, Borresen-Dale AL, et al:: Gene expression programs in response to hypoxia: cell type specificity and prognostic significance in human cancers. PLoS Med 2006, 3:e47.

28. The van de Vijver Lab [http://research.nki.nl/vandevijverlab/

29. Predicting a local recurrence after breast conserving therapy by gene expression profiling [http://microarray-pubs.stan ford.edu/wound local recurrence]

30. Chang HY, Sneddon JB, Alizadeh AA, Sood R, West RB, Montgomery K, Chi JT, Rijn Mv M, Botstein D, Brown PO: Gene expression signature of fibroblast serum response predicts human 
cancer progression: similarities between tumors and wounds. PLoS Biol 2004, 2:E7.

31. Tibshirani R, Hastie T, Narasimhan B, Chu G: Diagnosis of multiple cancer types by shrunken centroids of gene expression. Proc Natl Acad Sci USA 2002, 99:6567-6572.

32. Subramanian A, Tamayo $P$, Mootha VK, Mukherjee $S$, Ebert BL, Gillette MA, Paulovich A, Pomeroy SL, Golub TR, Lander ES, Mesirov JP: Gene set enrichment analysis: a knowledge-based approach for interpreting genome-wide expression profiles. Proc Natl Acad Sci USA 2005, 102:15545-15550.

33. Gene Set Enrichment Analysis: overview [http:// www.broad.mit.edu/gsea/]

34. lyer VR, Eisen MB, Ross DT, Schuler G, Moore T, Lee JC, Trent JM, Staudt LM, Hudson J Jr, Boguski MS, et al:: The transcriptional program in the response of human fibroblasts to serum. Science 1999, 283:83-87.

35. PAM: Prediction Analysis for Microarrays [http://wwwstat.stanford.edu/ tibs/PAM/index.html]

36. Ayers M, Symmans WF, Stec J, Damokosh Al, Clark E, Hess K, Lecocke M, Metivier J, Booser D, Ibrahim N, et al.: Gene expression profiles predict complete pathologic response to neoadjuvant paclitaxel and fluorouracil, doxorubicin, and cyclophosphamide chemotherapy in breast cancer. J Clin Oncol 2004, 22:2284-2293.

37. Chang JC, Wooten E, Tsimelzon A, Hilsenbeck S, Gutierrez MC, Elledge R, Mohsin S, Osborne CK, Chamness G, Allred DC, O'Connell P: Gene expression profiling for the prediction of therapeutic response to docetaxel in patients with breast cancer. Lancet 2003, 362:362-369.

38. Jansen MP, Foekens JA, van Staveren IL, Dirkzwager-Kiel MM, Ritstier K, Look MP, Meijer-van Gelder ME, Sieuwerts AM, Portengen $\mathrm{H}$, Dorssers LC, et al.: Molecular classification of tamoxifenresistant breast carcinomas by gene expression profiling. J Clin Oncol 2005, 23:732-740.

39. Michalides R, Griekspoor A, Balkenende A, Verwoerd D, Janssen L, Jalink K, Floore A, Velds A, van't Veer L, Neefjes J: Tamoxifen resistance by a conformational arrest of the estrogen receptor alpha after PKA activation in breast cancer. Cancer Cell 2004, 5:597-605.

40. Ma XJ, Wang Z, Ryan PD, Isakoff SJ, Barmettler A, Fuller A, Muir $B$, Mohapatra G, Salunga R, Tuggle JT, et al:: A two-gene expression ratio predicts clinical outcome in breast cancer patients treated with tamoxifen. Cancer Cell 2004, 5:607-616.

41. Paik S, Shak S, Tang G, Kim C, Baker J, Cronin M, Baehner FL, Walker MG, Watson D, Park T, et al:: A multigene assay to predict recurrence of tamoxifen-treated, node-negative breast cancer. N Engl J Med 2004, 351:2817-2826.

42. Vinh-Hung $V$, Verschraegen $C$ : Breast-conserving surgery with or without radiotherapy: pooled-analysis for risks of ipsilateral breast tumor recurrence and mortality. I Natl Cancer Inst 2004, 96:115-121.

43. Vrieling C, Collette L, Fourquet A, Hoogenraad WJ, Horiot JC, Jager JJ, Pierart M, Poortmans PM, Struikmans H, Van der Hulst M, et al: The influence of the boost in breast-conserving therapy on cosmetic outcome in the EORTC 'boost versus no boost' trial. EORTC Radiotherapy and Breast Cancer Cooperative Groups. European Organization for Research and Treatment of Cancer. Int J Radiat Oncol Biol Phys 1999, 45:677-685. 